\title{
High Frequency of Allelic Loss in Dysplastic Lichenoid Lesions
}

\author{
Lewei Zhang, Xing Cheng, Yong-hua Li, Catherine Poh, Tao Zeng, Robert Priddy, \\ John Lovas, Paul Freedman, Tom Daley, and Miriam P. Rosin
}

Faculty of Dentistry (LZ, Y-HL, CP, RP), University of British Columbia, and BC Cancer Research Centre (MPR), Cancer Control Unit, Vancouver, British Columbia, School of Kinesiology (XC, TZ, MPR), Simon Fraser University, Burnaby, British Columbia, Faculty of Dentistry (JL), Dalhousie University, Halifax, Nova Scotia, and Department of Pathology (TD), University of Western Ontario, London, Ontario, Canada; and The New York Hospital Medical Center of Queens (PF), Flushing, New York

SUMMARY: Oral lichen planus (OLP) is a common mucosal condition that is considered premalignant by some, whereas others argue that only lichenoid lesions with epithelial dysplasia are at risk of progressing into oral carcinoma. A recent study from this laboratory used microsatellite analysis to evaluate OLP for loss of heterozygosity (LOH) at loci on three chromosomal arms (3p, 9p, and 17p) (Am J Path 1997; Vol151:Page323-Page327). Loss on these arms is a common event in oral epithelial dysplasia and has been associated with risk of progression of oral leukoplakia to cancer. The data showed that, although dysplastic epithelium demonstrated a high frequency of LOH ( $40 \%$ for mild dysplasia), a significantly lower frequency of LOH was noted in OLP (6\%), which is even lower than that in hyperplasia (14\%). Such results do not support OLP as a lesion at risk for malignant transformation. As a second step of the research, we determined LOH frequencies in 61 dysplastic lichenoid lesions (mild 35; moderate 19; severe 7) using the same microsatellite markers and compared these results with data obtained from the first study and from 13 normal mucosal specimens. Dysplastic lichenoid lesions showed a high frequency of loss (54\% for lichenoid lesions with mild dysplasia), but values did not differ significantly from those observed in dysplasia of similar degree without lichenoid appearance. None of the normal mucosa demonstrated LOH. Epithelial dysplasia is a sign of malignant risk, independent of lichenoid changes. Such results suggest that pathologists should search for dysplasia carefully in lesions that otherwise qualify as OLP and that caution should be used when discounting dysplasia as being merely a reactive condition in lichenoid lesions. (Lab Invest 2000, 80:233-237).

$O$ ral lichen planus (OLP) is one of the most common oral mucosa diseases, occurring in approximately $1 \%$ of the general population (Pindborg et al, 1972; Scully and el-Kom, 1985). Unlike cutaneous lichen planus, OLP tends to be chronic. It has been suggested that complete remission of OLP is either nonexistent or infrequent (see review in Eisen 1993). Histologically, OLP is characterized by a dense bandlike lymphohistiocytic infiltrate in the immediate subepithelial region, with basal epithelial cell destruction.

There have been heated debates as to whether OLP per se is precancerous or whether only OLP-like lesions demonstrating epithelial dysplasia are potentially at risk of developing into cancer (Eisenberg and Krutchkoff, 1992; Holmstrup 1992; Krutchkoff and Eisenberg, 1985; Lovas et al, 1989). After retrospective analysis of original photomicrographs from a number of published cases of alleged malignant trans-

Received November 8, 1999.

This study was supported by the British Columbia Health Care Foundation and by the National Cancer Institute of Canada with funds from the Canadian Cancer Society.

Address reprint requests to: Dr. M. P. Rosin, School of Kinesiology, Simon Frazer University, Burnaby, British Columbia, Canada V5A ISR. Fax: 604-291-3040; E-mail: rosin@sfu.ca formation of OLP, Krutchkoff and Eisenberg concluded that many reported cases of oral carcinomas arising from OLP may have developed from lichenoid lesions with epithelial dysplasia (Eisenberg and Krutchkoff, 1992). As a result, the authors recommended that more strict criteria be applied in the diagnosis of OLP and that dysplastic lesions not be called OLP.

Are many lichenoid lesions with epithelial dysplasia called OLP? Data suggest that this may be the case. Dysplasia was reported in $11 \%$ of OLP $(n=100)$ by Urbizo-Velez et al (1990), and 25\% $(n=100)$ by De Jone et al (1984).

The presence and degree of epithelial dysplasia has been the histologic hallmark or gold standard for judging the malignant potential of preinvasive lesions. Why has it been widely ignored in these lichenoid lesions? There are two obvious reasons. First, the biopsies were not carefully examined for the presence of dysplasia because of the striking lichenoid features. An alternative explanation is that dysplasia was recognized but discounted as being due to changes in response to lymphohistiocytic infiltrate.

Inflammation is known to cause atypical epithelial changes resembling dysplasia and the atypia is believed to be reactive and not regarded as an indication 
of malignant potential. Such reactive changes are usually seen in the case of intense acute inflammation or mixed acute and chronic inflammation, typically near an ulcer. It is less clear whether such reactive changes readily occur in specific lichenoid dermatoses such as lichen planus and discoid lupus. Naturally, there is uncertainty about the significance of dysplasia when it is present in a lesion with striking lichenoid features that otherwise qualifies for the diagnosis of OLP. As a result, a lichenoid lesion with epithelial dysplasia may be signed out as dysplasia by one pathologist (who believes dysplasia is dysplasia regardless of lichenoid infiltrate) or as OLP by another pathologist (who overlooks the dysplasia or discounts the dysplasia as an inflammatory response).

Two important questions arise from these debates and disagreements: (a) Is OLP without dysplasia premalignant? (b) Is dysplasia still a sign of malignant potential in a lesion with striking lichenoid features? One way to answer these questions is to determine whether the genetic changes that commonly occur in early oral carcinogenesis (oral dysplasia) are found in OLP and dysplastic lichenoid lesions.

To answer the first question, a recent paper from this laboratory studied the molecular changes in OLP by microsatellite analysis (Zhang et al, 1997; editorial on the study in Allen, 1998). We chose this technique because of its sensitivity. Oral premalignant lesions are generally small with minute amounts of DNA. Microsatellite analysis requires only $5 \mathrm{ng}$ of DNA per reaction, but yields valuable data on a biologically significant event, the loss of chromosomal regions (loss of heterozygosity, LOH) that contain putative tumor suppressor genes (Rosin et al, in press; Califano et al, 1996). The study compared LOH at 3p, 9p, and $17 p$ in OLP (without dysplasia) with $\mathrm{LOH}$ in oral epithelial dysplasia and benign hyperplasia. Although dysplastic epithelium demonstrated a high frequency of LOH (40\% for mild dysplasia), a significantly lower frequency of LOH was noted in OLP (6\%), which is even lower than that in hyperplasia (14\%). Such results seem to indicate that OLP (without dysplasia) has no apparent malignant risk.

As a second step of the investigation, this study tested the hypothesis that epithelial dysplasia is a sign of malignant risk even when the lesion demonstrates striking lichenoid features. $\mathrm{LOH}$ at $3 p, 9 p$, and $17 p$ was analyzed in dysplastic lichenoid lesions. The results were compared with data obtained from the first study and from 13 normal mucosal specimens.

\section{Results}

Table 1 presents the mean age and smoking habits of individuals in the two study groups, together with similar data for the OLP and dysplasia cases from our previous study. A significantly higher number of smokers were found in patients with dysplasia (87.5\%) and dysplastic lichenoid lesions (77\%) compared with those patients with OLP (25\%) and normal epithelium $(25 \%)$ ( $p$ value varies from $p=0.0018$ to $p<0.0001$ ).

Table 2 presents LOH frequencies for the 61 dysplastic lichenoid lesions and the concurrently run samples of normal oral epithelium $(n=13)$. The data are presented as the percentage of cases in each group with $\mathrm{LOH}$ for loci on $3 p, 9 p$, or $17 \mathrm{p}$. In addition, a determination was made of the percentage of cases showing any allelic loss (3p, 9p, or $17 p)$, or with loss on more than one of these arms. Figure 1 represents a typical LOH analysis.

None of the samples with normal oral epithelium showed LOH. In contrast, a significant percentage of dysplastic lichenoid lesions demonstrated such loss (Table 2). LOH frequencies tended to increase with the severity of dysplasia in the lesions. High-grade dysplastic lichenoid lesions (those with severe dysplasia or carcinoma in situ, CIS) contained higher $\mathrm{LOH}$ frequencies than low-grade dysplastic lichenoid lesions (those with mild and moderate dysplasia) $\left(\chi^{2}\right.$ test for trend, $p=0.0489$ ).

Of the 61 patients with dysplastic lichenoid lesions, seven patients had biopsies from lesions from two geographic locations in the oral cavity. All of these biopsies showed intense submucosal lymphocytic infiltrate. Six of seven patients had dysplasia in both biopsied lichenoid lesions and one patient had one dysplastic lichenoid lesion and one OLP. Information on smoking habits was available for five of the seven patients; of these five patients, four were smokers.

\section{Discussion}

Although OLP is considered a premalignant lesion by the World Health Organization (see review in Holmstrup, 1992), it is also argued that OLP per se is not precancerous and that only OLP-like lesions demonstrating dysplasia are potentially at risk of developing into cancer (Eisenberg and Krutchkoff, 1992; Krutchkoff \& Eisenberg, 1985; Lovas et al, 1989). A recent study from this laboratory has shown that OLP (without dysplasia) lacked the characteristic genetic alter-

Table 1. Smoking Habit and Age of Patients with Oral Lesions

\begin{tabular}{lcccc}
\hline & & \multicolumn{2}{c}{ Number of patients with known habit (\%) } \\
\cline { 4 - 5 } \multicolumn{1}{c}{ Diagnosis } & Number of cases & Mean age (years) & Smoker (present or past) & Non-smoker \\
\hline Oral licheu planus & 33 & 47 & $8(25 \%)$ & $24(75 \%)$ \\
Dysplasia & 37 & 60 & $21(87.5 \%)$ & $3(12.5 \%)$ \\
Dysplastic lichenoid lesion & 61 & 53 & $30(77 \%)$ & $9(23 \%)$ \\
Normal & 13 & 42 & $3(25 \%)$ & $9(75 \%)$ \\
\hline
\end{tabular}


Table 2. Loss of Heterozygosity in Normal Epithelium and Lichenoid Lesions with Dysplasia ${ }^{a}$

\begin{tabular}{|c|c|c|c|c|c|c|}
\hline \multirow[b]{2}{*}{ Diagnosis } & \multirow{2}{*}{$\begin{array}{l}\text { Number } \\
\text { of cases }\end{array}$} & \multicolumn{5}{|c|}{ Total allelic loss/informative cases ( $\%$ ) } \\
\hline & & $3 p$ & $9 p$ & $17 p$ & Any loss & $>1$ loss \\
\hline Normal & 13 & 0 & 0 & 0 & 0 & 0 \\
\hline Lichenoid lesions with mild dysplasia & 35 & $9 / 33(27)$ & $14 / 35(40)$ & $4 / 33(12)$ & $19 / 35(54)$ & 6/33 (18) \\
\hline Lichenoid lesions with moderate dysplasia & 19 & $5 / 17(29)$ & $6 / 18(33)$ & $5 / 19(26)$ & $10 / 19(53)$ & $5 / 19(26)$ \\
\hline Lichenoid lesions with severe dysplasia or CIS & 7 & $3 / 7 \quad(43)$ & $6 / 7 \quad(86)$ & $3 / 7 \quad(43)$ & $6 / 7 \quad(86)$ & $5 / 7 \quad(71)$ \\
\hline
\end{tabular}

${ }^{a}$ Values given as number of samples showing loss/total number of informative cases (\% of cases in parentheses).

CIS, carcinoma in situ.

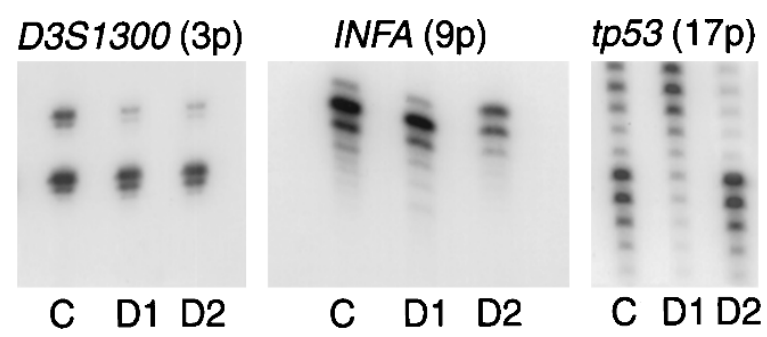

Figure 1.

Microsatellite analysis of 2 mildly dysplastic lichenoid lesions (D1 and D2) identified in concurrent biopsies from different oral sites in one patient. DNA was isolated from cells microdissected from the stroma $(C)$, as a source of normal DNA, and from each dysplastic lichenoid lesion. Microsatellite markers and the chromosomal arm being assayed are indicated above each block. Both lesions (D1 and D2) showed loss of the upper allele at D3S1300 on 3p. In contrast, biopsy D2 showed a loss of the lower allele at INFA on 9p and biopsy $D 1$ showed retention of both alleles at this locus. Furthermore, one of the dysplastic lichenoid lesions (D1) showed loss of the lower allele at TP53 on $17 p$, whereas the other (D2) had a loss of the upper allele.

ations (loss of tumor suppressor genes) commonly seen in oral premalignant lesions (Zhang et al, 1997). In contrast to these results, the present study has shown that dysplastic lichenoid lesions contain a high frequency of $\mathrm{LOH}$, with values that did not differ significantly from those observed among dysplastic lesions of similar degree without lichenoid appearance: $\mathrm{LOH}$ at any arm was noted in 54\% mildly dysplastic lichenoid lesions compared with $40 \%$ of mild dysplasia ( $p=0.4908$ ); 53\% moderately dysplastic lichenoid lesions compared with $46 \%$ of moderate dysplasia $(p=1)$; and $86 \%$ of lichenoid lesions with severe dysplasia or CIS compared with $81 \%$ in severe dysplasia/CIS ( $p=1$ ) (Zhang et al, 1997). Similar to dysplasia (without lichenoid mucosites), dysplastic lichenoid lesions showed a significantly higher frequency of $\mathrm{LOH}$ when compared with hyperplastic lesions, even when the comparison was made between mildly dysplastic lichenoid lesions and hyperplasias (any LOH: $54 \%$ versus $14 \%, p=0.0014 ;>1$ loss: $18 \%$ versus $0 \%, p=0.0257$ ) (Table 2; Zhang et al, 1997).

Such high frequencies of loss in chromosome regions containing presumptive tumor suppressor genes would suggest that the presence of epithelial dysplasia is a sign of malignant risk, independent of lichenoid changes. The results suggest that atypical epithelial changes in the presence of lichenoid mucosites is more likely to represent true dysplastic changes and should not be discounted as reactive change, which is more commonly seen in the case of intense acute or mixed acute and chronic inflammation typically near an ulcer. In view of the fact that there is some tendency for pathologists to miss dysplasia in a lesion otherwise qualifying for the diagnosis of OLP, or to discount dysplasia as reactive in such lesions, we would echo the caution from Krutchkoff and Eisenberg that more strict criteria should be used in diagnosing OLP and more attention should be paid to examining lichenoid lesions for dysplasia (Eisenberg and Krutchkoff, 1992).

Because dysplastic lichenoid lesions resemble both OLP and dysplasia, one would question whether there is any relationship between OLP and dysplasia. There may be two scenarios. In many cases, the dysplastic lichenoid lesion may represent a dysplasia de novo and the lesion may have no relation to OLP. This is supported by the fact that oral epithelial dysplasia with varying degrees of submucosal lymphocytic infiltrate is a common phenomenon, although a striking lichenoid infiltrate suggestive of OLP (cases used in this study) is less frequent. Such clinically white lesions are more likely leukoplakias rather than OLP, especially when they present as single plaque-like lesions.

On the other hand, a relationship may indeed exist between OLP and dysplasia in some cases and the dysplastic lichenoid lesions may actually represent OLP that have undergone dysplastic changes. In the previous study, we have shown that one patient (a smoker) with both OLP and a dysplastic lichenoid lesion demonstrated LOH only in the dysplastic lichenoid lesion. We hypothesized that this patient originally had only OLP (multiple lesions at different sites), and subsequently, one lesion, possibly due to tobacco exposure, underwent dysplastic changes and LOH. In this study of 61 patients with dysplastic lichenoid lesions, 7 had multiple biopsies available for histology and $\mathrm{LOH}$ analysis. The presence of multiple white lesions clinically, the presence of dense submucosal lymphohistiocytic infiltrates in these multiple lesions histologically, and the presence of OLP in one of the patients would suggest an OLP process. The majority (4 of 5) of these patients with known habits were smokers. Again one could hypothesize that these dysplastic lichenoid lesions may have been originally OLP which, under the influence of oral carcinogens, underwent dysplastic changes. Of interest, in 5 of the 7 cases that had multiple biopsies, a different pattern of loss was observed in the different lesion sites (see 
Fig. 1, for example). The presence of independent mutations at separate lichenoid regions suggests these inflamed sites may be susceptible to genetic changes. However, the only convincing way of proving that OLP develop into dysplastic lichenoid lesions would be to undertake prospective studies and to demonstrate that clinically and histologically confirmed OLP cases become dysplastic. Similarly the only convincing way of proving that lichenoid lesions in a smoker have a higher chance of malignant transformation than the rest of similarly smoking-exposed oral mucosa would again be through prospective studies.

In summary, the results of the study suggest that lichenoid lesions should be carefully examined for the presence of dysplastic changes, because such alterations often contain genetic changes associated with malignant risk. Furthermore, the presence of epithelial dysplastic changes in lichenoid lesions should not be readily discounted as reactive, as in the case of intense acute inflammation and ulceration. Finally, the study results suggest that patients with OLP should all be under periodic observation by qualified personnel, especially if they are smokers, to ensure that clinical and histologic evidence of premalignant and malignant changes are detected.

\section{Materials and Methods}

\section{Tissues and DNA Extraction}

Most of the specimens were selected from archival paraffin blocks obtained from the Division of Oral Pathology at Vancouver Hospital and Health Sciences Centre, Vancouver, Canada. Some specimens came from oral pathology biopsy services in other North American Institutes. Two groups of lesions were used: dysplastic lichenoid lesions and specimens with normal oral epithelium. The first group consisted of lesions with epithelial dysplasia as well as striking lichenoid features. The criteria used for the diagnosis of dysplastic lichenoid lesions were those described by Krutchkoff and Eisenberg (1985). The latter group consisted of biopsies with unremarkable histology, amalgam tattoo, melanotic macules, and vascular lesions (varicose vein and hemangioma). Histologic diagnoses of the specimens were reconfirmed by two of the authors ( $\mathrm{LZ}$ and RP), both oral pathologists.

All lesions were microdissected by LZ. Connective tissue from each specimen was submitted as a source of normal DNA. The dissected tissues were placed in sodium dodecyl sulfate/proteinase $\mathrm{K}$ at $48^{\circ} \mathrm{C}$ and spiked twice a day for 72 hours with fresh proteinase K. Genomic DNA was extracted with phenolchloroform and precipitated with ethanol as previously described (Zhang et al, 1997).

\section{LOH Assay}

DNA was analyzed for $\mathrm{LOH}$ by using microsatellite markers (Research Genetics, Huntsville, Alabama) that mapped to the following regions: 3p14.2 (D3S1234, D3S1300), 3p25.3-25.1 (D3S1110), 9p21 (IFNA,
D9S171, D9S1751, D9S1748), 17p13.1 (TP53), and 17p11.1-12 (CHRNB1). These markers are localized in regions previously shown to be frequently lost in head and neck tumors. The 9p21 locus has been linked to a putative tumor suppressor $\mathrm{p} 16^{\mathrm{INK} 4 \mathrm{~A}}$, a gene that codes for a cyclin-dependent kinase inhibitor involved in regulation of the cell cycle (Kamb et al, 1994). Three regions of loss have been identified for $3 p$ (3p14, 3p21.3, and 3p24) (Maestro et al, 1993). We chose to focus primarily on the 3p14 locus because $\mathrm{LOH}$ at this region has not only been reported high in head and neck squamous cell carcinoma (SCC) (Mao et al, 1996a; 1996b; Virgilio et al, 1996), but also has been recently shown to be associated with the risk of progression of oral premalignant lesions to SCC (Mao et al, 1996a; 1996b). We did, however, include one marker in the more telomeric region of loss for comparison (D3S1110). Finally, the study included a marker located within the p53 gene (TP53), as well as one at 17p11.1-p12 (CHRNB1) because this locus is sometimes lost in the absence of LOH at TP53 (Adamson et al, 1994). Although the putative suppressor gene in this region is believed to be $\mathrm{p} 53$, there is some suggestion that a second tumor suppressor gene could be present in this region (Adamson et al, 1994).

The protocol used for $\mathrm{LOH}$ analysis is described in Zhang et al (1997). After PCR amplification, PCR products were separated on $7 \%$ urea-formamidepolyacrylamide gels and visualized by autoradiography. Samples were coded and LOH was scored without knowledge of diagnosis. For informative cases, allelic loss was inferred when the signal intensity of one allele was at least $50 \%$ decreased in the DNA sample from a lesion, compared with the corresponding allele in the matching connective tissue DNA. All samples showing allelic loss were subjected to repeat analysis after a second independent amplification.

\section{Statistical Analysis}

Groups were compared with the Fisher exact test (two-tailed) and $\chi^{2}$ test for trend. All $p$ values were two-sided. A $p$ value of 0.05 or less was considered significant.

\section{References}

Adamson R, Jones AS, and Field JK (1994). Loss of heterozygosity studies on chromosome 17 in head and neck cancer using microsatellite markers. Oncogene 9:2077-2082.

Allen CM (1998). Is lichen planus really premalignant? (Editorial). Oral Surg Oral Med Oral Path 85(4):347.

Califano J, van der Riet P, Westra W, Nawroz H, Clayman G, Piatadosi S, Corio R, Lee D, Greenberg B, Koch W, and Sidransky D (1996). Genetic progression model for head and neck cancer: Implications for field cancerization. Cancer Res 56:2488-2492.

De Jone WFB, Albrecht M, Banoczy J, and Van Der Waal I (1984). Epithelial dysplasia in oral lichen planus. Int J Oral Surg 13:221-225. 
Eisen D (1993). The therapy of oral lichen planus. Crit Rev Oral Biol Med 4:141-158.

Eisenberg E and Krutchkoff DJ (1992). Lichenoid lesions of oral mucosa. Oral Surg 73:699-704.

Holmstrup P (1992). The controversy of a premalignant potential of oral lichen planus is over. Oral Surg 73:704-706.

Kamb A, Gruis NA, Weaver-Feldhaus J, Liu Q, Harshman K, Tavtigian SV, Stockert E, Day RS, III, Johnson BE, and Skolnick MH (1994). A cell cycle regulator potentially involved in the genesis of many tumor types. Science 264:436-440.

Krutchkoff DJ and Eisenberg E (1985). Lichenoid dysplasia. A distinct histopathologic entity. Oral Surg 30:308-315.

Lovas JGL, Harsanyi BB, and ElGeneidy AK (1989). Oral lichenoid dysplasia: A clinicopathologic analysis. Oral Surg 68:57-63.

Maestro R, Gasparotto D, Vukosavljevic T, Barzan L, Sulfaro S, and Boiocchi M (1993). Three discrete regions of deletion at $3 p$ in head and neck cancers. Cancer Res 53:5775-5779.

Mao L, Fan Y-H, Lotan R, and Hong WK (1996a). Frequent abnormalities of FHIT, a candidate tumor suppressor gene, in head and neck cancer cell lines. Cancer Res 56:5128-5131.

Mao L, Lee JS, Fan YH, Ro JY, Batsakis JG, Lippman S, Hittleman W, and Hong WK (1996b). Frequent microsatellite alterations at chromosomes 9p21 and 3p14 in oral premalignant lesions and their value in cancer risk assessment. Nature Med 2:682-685.
Pindborg JJ, Mehta FS, Daftary DK, Gupta PC, and Bhousle RB (1972). Prevalence of oral lichen planus among 7639 Indian villagers in Kerala, South India. Acta Derm Venereol (Stockh) 52:216-20.

Rosin MP, Cheng X, Poh C, Lam WL, Huang Y, Lovas J, Berean K, Epstein JB, Priddy R, Le ND, and Zhang L (In press, 2000). Use of allelic loss to predict malignant risk for low-grade oral epithelial dysplasia. Clin Cancer Res.

Scully C and el-Kom M (1985). Lichen planus: Review and update on pathogenesis. J Oral Path 14:431-58.

Urbizo-Velez J, Perez IR, Albrecht M, and Banoczy J (1990). Comparative histopathological studies in oral lichen planus. Acta Morphologica Hungarica 38:71-81.

Virgilio L, Shuster M, Gollin SM, Veronese ML, Ohta M, Huebner K, and Croce CM (1996). FHIT gene alterations in head and neck squamous cell carcinomas. Proc Natl Acad Sci USA 93:9770-9775.

Zhang L, Michelsen C, Cheng X, Zeng T, Priddy R, Rosin MP (1997). Molecular analysis of oral lichen planus: Is this a premalignant lesion? Am J Path 151(2):323-327. 